\title{
A second RGD motif in the 1D capsid protein of a SAT1 type foot-and-mouth disease virus field isolate is not essential for attachment to target cells
}

\author{
P. Storey ${ }^{\mathrm{a}, \mathrm{b}}$, J. Theron ${ }^{\mathrm{b}}$, F.F. Maree ${ }^{\mathrm{a}}$ and H.G. O'Neill ${ }^{\mathrm{a}}$ \\ ${ }^{\mathrm{a} O n d e r s t e p o o r t ~ V e t e r i n a r y ~ I n s t i t u t e, ~ E x o t i c ~ D i s e a s e s ~ D i v i s i o n, ~ P r i v a t e ~ B a g ~ X 05, ~}$ \\ Onderstepoort 0010, South Africa \\ ${ }^{b}$ Department of Microbiology and Plant Pathology, University of Pretoria, Pretoria \\ 0002, South Africa
}

\section{Abstract}

The amino acid sequence motif Arg-Gly-Asp (RGD), located in the surface-exposed $\beta \mathrm{G}$ $\beta \mathrm{H}$ loop of the 1D protein of different serotypes and subtypes of foot-and-mouth disease virus (FMDV), is highly conserved and participates in binding of FMDV to susceptible cells. Previous sequence analyses of the 1D-encoding region of a FMDV serotype SAT1 field isolate from Namibia (NAM/307/98) indicated the presence of a second RGD motif upstream of the conserved $\beta \mathrm{G}-\beta \mathrm{H}$ loop RGD. The role of these RGD sequences in virus infection was investigated by mutating the $\beta \mathrm{G}-\beta \mathrm{H}$ loop RGD to a KGE tripeptide, using a genome-length infectious chimeric cDNA clone. Although the infectivity of the derived mutant viruses for baby hamster kidney cells (BHK-21) was lost, subsequent replacement of the KGE sequence with RGD in the mutant cDNA clone led to recovery of infectious viruses. Furthermore, viral RNA replication could be demonstrated with the genetically engineered mutant and non-mutant viruses. The presence of virus particles in the transfected cells could be also demonstrated by electron microscopy. These results demonstrate that, in contrast to the $\beta \mathrm{G}-\beta \mathrm{H}$ loop RGD motif, the second RGD sequence in 
the capsid protein 1D of NAM/307/98 does not function as a ligand for receptor binding in BHK-21 cells.

\section{Article Outline}

1. Introduction

2. Materials and methods

2.1. Cell lines, viruses and plasmids

2.2. RNA isolation, cDNA synthesis, PCR amplification and nucleotide sequencing

2.3. Construction of genome-length chimeric and mutant chimeric cDNA clones

2.4. In vitro RNA synthesis, transfection and virus recovery

2.5. Detection of RNA replication

2.6. Electron microscopy

3. Results

3.1. Location of the second RGD motif in the $1 \mathrm{D}$ capsid protein of the parental NAM/307/98 field isolate

3.2. Receptor binding properties of the SAT1 type FMDV field isolate NAM/307/98

3.3. Infectivity of the chimeric and mutant chimeric NAM/SAT2 viruses

3.4. Detection of viral RNA replication and production of chimeric and mutant chimeric NAM/SAT2 viruses in BHK-21 cells

4. Discussion

Acknowledgements

References

\section{Introduction}

Foot-and-mouth disease (FMD), of which foot-and-mouth disease virus (FMDV) is the causative agent, is an economically important and highly contagious disease of clovenhoofed animals, most notably cattle, pigs and sheep, as well as several wild-life species (Thomson et al., 2003 and Thomson and Bastos, 2004). The seven serotypes of FMDV (types O, A, C, Asia1, and the South African Territories [SAT] types 1, 2, and 3) are 
members of the Aphthovirus genus of the family Picornaviridae (Grubman and Baxt, 2004). The FMDV virion consists of an icosahedral shell composed of 60 copies each of four structural proteins, $1 \mathrm{~A}$ to $1 \mathrm{D}$, surrounding a single-stranded, positive-sense RNA. Capsid proteins 1B to 1D form the outer capsid surface, whilst 1A is located at the inner capsid surface of the virion. The capsid protein 1D contains a mobile loop between the $\beta \mathrm{G}$ and $\beta \mathrm{H}$ strands on the virus surface that not only contains the major immunodominant epitopes of the virion, but also a highly conserved arginine-glycine-aspartic acid (RGD) sequence (Acharya et al., 1989, Logan et al., 1993 and Mateu, 1995).

The RGD tripeptide is a major determinant in the interaction of a number of protein ligands with cell surface receptors of the integrin superfamily (D'Souza et al., 1991). Several studies, using different approaches, have indicated that naturally occurring isolates of FMDV attach to cells via the highly conserved, surface-exposed RGD motif (Fox et al., 1989, Baxt and Becker, 1990 and Mason et al., 1994). FMDV has subsequently been reported to utilize multiple RGD-dependant integrins of the $\alpha_{\mathrm{v}}$ subgroup to initiate infection, including $\alpha_{v} \beta_{3}, \alpha_{v} \beta_{6}, \alpha_{v} \beta_{1}$ and $\alpha_{v} \beta_{8}$ (Berinstein et al., 1995, Jackson et al., 2000b, Jackson et al., 2002 and Jackson et al., 2004). However, FMDV can also enter cells through non-integrin-mediated pathways (Mason et al., 1993 and Baxt and Mason, 1995). Tissue culture-adapted viruses can use heparan sulfate, a cell surface glycosaminoglycan, as a receptor to enter cells (Jackson et al., 1996 and Sa-Carvalho et al., 1997). More recently, it has become apparent that FMDV may also use receptors of unknown identity that are neither integrin nor heparan sulfate (Martinez et al., 1997, Baranowski et al., 2000 and Zhao et al., 2003), thus indicating the existence of other receptor molecules and alternative mechanisms for FMDV entry into cells. Here we present studies on a SAT1 type FMDV that was isolated from an African buffalo (Syncerus caffer) in the West Caprivi Game Reserve, Namibia, in 1998 (referred to as $\mathrm{NAM} / 307 / 98$ ). Nucleotide characterization of the 1D-encoding region of NAM/307/98 indicated the presence of a second RGD triplet located upstream of the $\beta \mathrm{G}-\beta \mathrm{H}$ loop RGD (Bastos et al., 2001). Due to the novel occurrence of two RGD motifs in capsid protein 1D of NAM/307/98, the role of these motifs in receptor binding and cell infection was determined by introducing mutations within the conserved $\beta \mathrm{G}-\beta \mathrm{H}$ loop RGD sequence via a genome-length infectious chimeric cDNA clone. Following in vitro transcription 
and transfection of BHK cells with the mutated RNAs, the genetically engineered viruses were examined for their infectious potential.

\section{Materials and methods}

\subsection{Cell lines, viruses and plasmids}

Baby hamster kidney 21 cells (BHK-21, ATCC CCL-10) were maintained in Eagle's basal medium (BME; Invitrogen) containing 10\% fetal calf serum (FCS; Delta Bioproducts) and 10\% tryptose phosphate broth (Sigma). Instituto Biologico Renal Suino-2 cells (IB-RS-2) were maintained in RPMI medium (Sigma) and Chinese hamster ovary cells strain K1 (CHO-K1, ATCC CCL-61) were maintained in Ham's F-12 medium (Invitrogen), both supplemented with 10\% FCS. Plaque assays were performed using a tragacanth overlay method (Grubman et al., 1979) and 1\% methylene blue staining (Van Rensburg et al., 2004). The origin of SAT1/NAM/307/98 (passage history: primary pig kidney passage 1; IB-RS-2 passage 1) and SAT2/ZIM/7/83 (passage history: bovine passage 1; BHK passage 5; bovine passage 1; BHK passage 3) has been described elsewhere (Bastos et al., 2001 and Van Rensburg et al., 2004). pSAT2, a previously constructed genome-length infectious cDNA clone of ZIM/7/83 (Van Rensburg et al., 2004), was used as backbone in the construction of chimeric cDNA clones.

\subsection{RNA isolation, cDNA synthesis, $P C R$ amplification and nucleotide sequencing}

RNA was extracted from infected tissue culture samples using a guanidinium-based nucleic acid extraction method (Bastos, 1998) and used as template for cDNA synthesis. Avian myeloblastosis virus reverse transcriptase (Promega), random hexamers (Roche) and oligonucleotide 2B (5'-GAC ATG TCC TCC TGC ATC TG-3') (Vangrysperre and De Clercq, 1996) were used for reverse transcription, which was carried out for $1 \mathrm{~h}$ at $42{ }^{\circ} \mathrm{C}$. The external capsid-coding region of NAM/307/98 was amplified using the Expand ${ }^{\mathrm{TM}}$ High Fidelity PCR system (Roche) and flanking oligonucleotides, cDNANAM and cDNA-2A (see Section 2.3). The nucleotide sequence of the amplicon was determined using selected oligonucleotides and the ABI PRISM ${ }^{\mathrm{TM}}$ BigDye $^{\mathrm{TM}}$ Terminator Cycle Sequencing Ready Reaction kit v3.0 (Perkin-Elmer Applied Biosystems), followed by resolution on an ABI PRISM 310 Genetic Analyser (Applied Biosystems). The 
nucleotide sequence of the capsid-encoding region of NAM/307/98 has been submitted to GenBank under the accession number AY770519.

\subsection{Construction of genome-length chimeric and mutant chimeric cDNA clones}

The external capsid-coding region of NAM/307/98 was PCR-amplified using oligonucleotides cDNA-NAM (5'-C GGA ATA TTG ACC ACC AGC CAT GGT ACC ACC AC-3'; SspI site underlined) and cDNA-2A (5'-CGC CCC GGG GTT GGA CTC AAC GTC TCC-3'; XmaI site underlined). The PCR amplicon was digested and ligated into pSAT2, of which the corresponding external capsid-encoding region had been removed using the same endonucleases, to generate pNAM/SAT2. Whereas the SspI site introduced an amino acid change (Met [ATG] to Leu [TTG], indicated in italics in cDNA-NAM) at codon 15 of protein $1 \mathrm{~B}$, the XmaI site was added by silent mutation. Site-directed mutagenesis of the 1D $\beta$ G- $\beta$ H loop RGD sequence of NAM/307/98 to KGE was carried out using a "megaprimer" method in which three primers and two PCRs were used (Landt et al., 1990). Briefly, the first PCR reaction was performed utilizing pNAM/SAT2 as template, and NAMmut 1 (5'-C GAG AAC ATT aaa GGT GAg CTT GCA ACG C-3'; lower cased letters represent altered bases) as the sense mutagenic and cDNA-2A as the antisense oligonucleotides. The 270-bp amplicon was used as a "megaprimer" along with the 5'-specific sense oligonucleotide cDNA-NAM in a second PCR reaction. Cycling conditions for both PCRs were as follows: $95^{\circ} \mathrm{C}$ for $20 \mathrm{~s}, 55^{\circ} \mathrm{C}$ for $20 \mathrm{~s}$, and $72{ }^{\circ} \mathrm{C}$ for $30 \mathrm{~s}$ ( 25 cycles). The resulting 2.2-kb DNA fragment was digested with SspI and XmaI and inserted into the corresponding sites of pNAM/SAT2 to yield pNAMKGE/SAT2. Substitution of codons specifying KGE for the natural RGD codons was accomplished by the same procedure, except that pNAMKGE/SAT2 was used as template and NAMmut3 (5'-C GAG AAC ATT cgc GGT GAc CTT GCA ACG C-3'; lower cased letters represent altered bases) was used as the mutagenic oligonucleotide. The amplicon was inserted into the corresponding sites of pNAMKGE/SAT2, as described above, to yield pNAMRGD/SAT2. In all instances, the alterations in the recombinant clones were verified by sequence analysis. 


\subsection{In vitro RNA synthesis, transfection and virus recovery}

The constructed chimeric cDNA clones and pSAT2 were linearized at the SwaI and NotI sites, respectively, following the poly $(\mathrm{A})$ tract, and were used as templates to synthesize RNA in vitro with the MEGAScript ${ }^{\circledR}$ T7 kit (Ambion) according to the manufacturer's instructions. RNA transcripts $(3 \mu \mathrm{g})$ were introduced into BHK-21 cells, seeded in $35 \mathrm{~mm}$ diameter tissue culture plates, using Lipofectamine $2000^{\mathrm{TM}}$ reagent (Invitrogen) according to the manufacturer's instructions. Transfected monolayers were incubated at $37{ }^{\circ} \mathrm{C}$ with $5 \% \mathrm{CO}_{2}$ for $24 \mathrm{~h}$ in BME containing $1 \% \mathrm{FCS}$ and $25 \mathrm{mM}$ HEPES. The supernatants were used to infect fresh BHK cell monolayers and incubated for up to $48 \mathrm{~h}$ at $37^{\circ} \mathrm{C}$. Viruses were subsequently harvested by freeze-thawing and passed three more times on fresh BHK cell monolayers, using $10 \%$ of the supernatants of previous passages, as described before (Van Rensburg et al., 2004).

\subsection{Detection of RNA replication}

The culture supernatants of transfected BHK cell monolayers and of cell monolayers used in the first virus passage were collected and clarified by centrifugation at $2000 \mathrm{rpm}$ for $5 \mathrm{~min}$. The transfected cell pellets were rinsed twice with $3 \mathrm{ml}$ BME medium, suspended in $1 \mathrm{ml} \mathrm{BME}$ and lysed by a single freeze-thaw cycle. To remove cell-associated extracellular viral particles from cells used in the first passage, the cells were rinsed once with $3 \mathrm{ml}$ MBS buffer (1 M MES, 1.45 M NaCl, pH 5.5) (Curry et al., 1995) and then twice with $3 \mathrm{ml} \mathrm{BME}$ medium prior to lysis in $3 \mathrm{ml} \mathrm{BME}$, as described above. Cell monolayers used in subsequent rounds of passaging were lysed by freeze-thawing without separating the culture supernatant and cells. To degrade endogenous cellular RNA and unpackaged viral RNA, an aliquot (10\%) of each of the prepared samples was incubated at room temperature for $45 \mathrm{~min}$ with $100 \mu \mathrm{g}$ RNase A (Roche) and $1 \times 10^{6} \mathrm{U}$ RNase T1 (Roche). Following inhibition of the RNase enzymes by the addition of $1 \times \mathrm{TE}$ buffer (10 mM Tris-HCl, 2 mM EDTA, pH 7.4) containing $20 \mathrm{U}_{\mathrm{RNasin}}{ }^{\circledR}$ ribonuclease inhibitor (Promega), the 1D genomic region of the recovered chimeric viruses, inclusive of the sequence encoding the two RGD motifs, was PCR-amplified and sequenced. For this purpose, RNA was extracted from the RNase-treated samples using TRIzol (GibcoBRL), reverse transcribed and then amplified using Taq DNA polymerase (Roche) as 
described previously (Bastos, 1998). The nucleotide sequence of the gel-purified amplicons was determined as described above.

\subsection{Electron microscopy}

BHK-21 monolayers, transfected with in vitro synthesized mutant and non-mutant chimeric RNAs, were lysed at $24 \mathrm{~h}$ post-transfection by the addition of $0.1 \%$ Triton X100 and EDTA ( $\mathrm{pH} 8$ ) to a final concentration of $20 \mathrm{mM}$. Cell lysates were centrifuged to remove cellular debris (4500 rpm; $10 \mathrm{~min}$ ). The supernatants were placed on a sucrose density gradient $(25,35$ and $60 \%$, w/v sucrose) and ultracentrifuged at 35,000 rpm for $18 \mathrm{~h}$ at $20^{\circ} \mathrm{C}$ in a SW55 rotor (Beckman Coulter). Virus particles were removed from the interface formed by the $35-60 \%$ sucrose layers, concentrated by ultracentrifugation at $45,000 \mathrm{rpm}$ for $16 \mathrm{~h}$ and suspended in TNE buffer (10 mM Tris (pH 7.5), $150 \mathrm{mM} \mathrm{NaCl}$, $2 \mathrm{mM}$ EDTA). The virus particles were viewed by electron microscopy after negative staining with $2 \%$ uranyl acetate in a Jeol1200EX MkI microscope at $80 \mathrm{kV}$.

\section{Results}

\subsection{Location of the second RGD motif in the $1 D$ capsid protein of the parental NAM/307/98 field isolate}

Analysis of the deduced amino acid sequence of the capsid-encoding region of the NAM/307/98 field isolate indicated the presence of a conserved RGD sequence (amino acid residues 149-151) located in a hypervariable region of the 1D capsid protein, as well as a second, upstream RGD sequence located at residues 110-112 (Fig. 1A). This second RGD motif was stably retained upon passage in IB-RS-2 cells (PK1RS1) and was identical in sequence to that reported by Bastos et al. (2001), following primary isolation of the virus in pig kidney cells (PK1). In all FMDV serotypes, the $\beta \mathrm{G}-\beta \mathrm{H}$ loop RGD sequence is highly exposed to the surface (Baxt and Becker, 1990, Parry et al., 1990, Mason et al., 1994 and Jackson et al., 1997). To determine the location of the second, non- $\beta \mathrm{G}-\beta \mathrm{H}$ loop RGD sequence on the viral capsid, the $1 \mathrm{D}$ capsid protein of $\mathrm{NAM} / 307 / 98$ was aligned with that of type $\mathrm{O}_{1} \mathrm{BFS}$ using CLUSTALW. Whereas the RGD sequence located in the $\beta G-\beta H$ loop was conserved, the upstream RGD sequence in $\mathrm{NAM} / 307 / 98$ aligned with a KAP sequence in type $\mathrm{O}_{1} \mathrm{BFS}$ (Fig. 1A). Based on the 
previously elucidated crystal structure of the type $\mathrm{O}_{1}$ BFS virion (Logan et al., 1993), the KAP sequence is located on a short surface-exposed loop between $\beta F$ and $\beta G$ (Fig. 1B). Examination of the complete virion reveals that the surface-exposed KAP sequence is visible surrounding the five-fold axes located distant from the $\beta G-\beta H$ loop RGD motif (Fig. 1C). This is in agreement with previous reports that indicated that the short surfaceexposed loop between $\beta F$ and $\beta G$ is situated at the five-fold axes of symmetry (Acharya et al., 1990 and Fry et al., 1990). These results suggested that the second RGD motif in the $1 \mathrm{D}$ capsid protein of NAM/307/98 is likewise located on the protein surface and thus could play a role in cell entry.
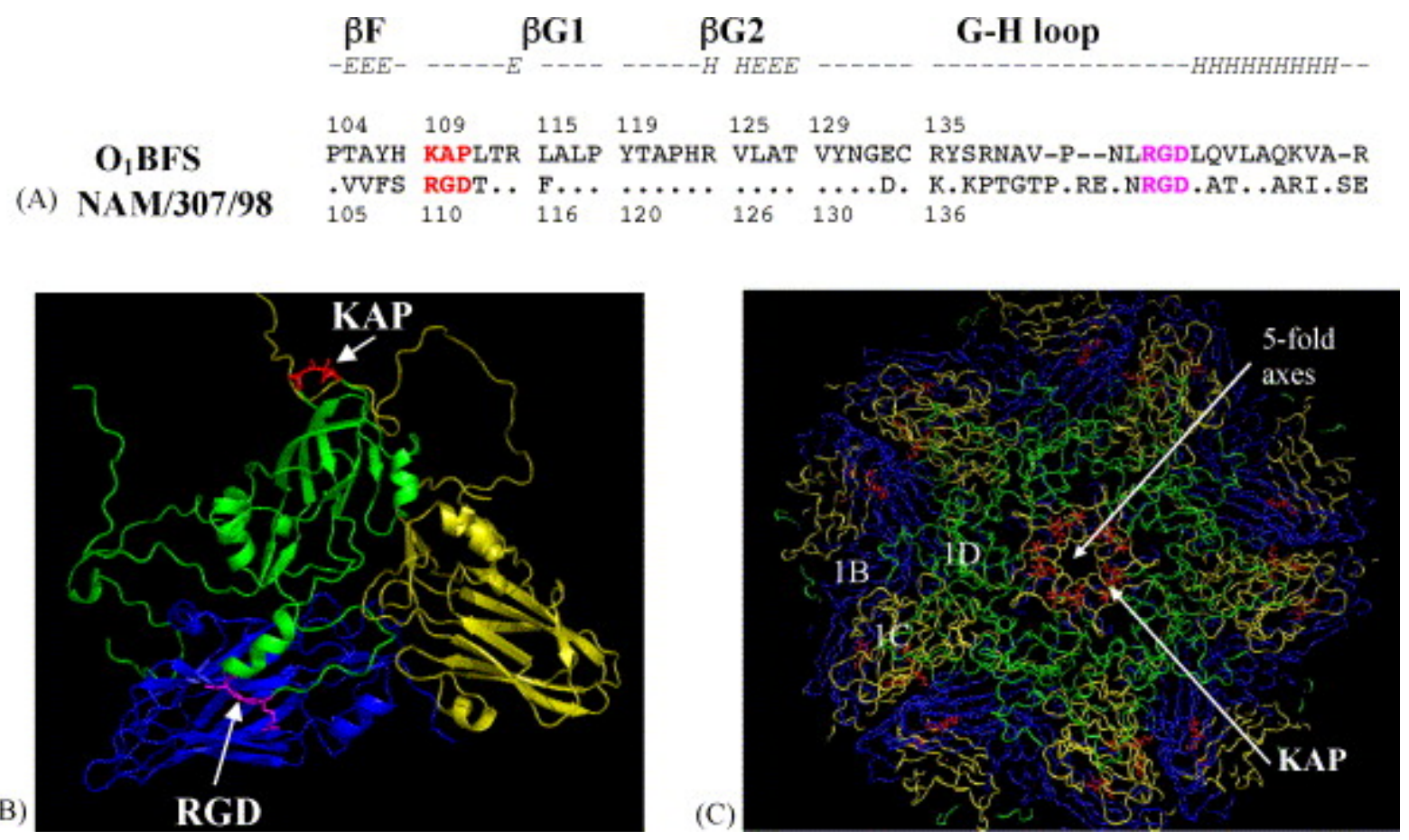

Fig. 1. Putative location of two RGD sequences identified in the 1D capsid protein of NAM/307/98. (A) Amino acid sequence alignment (adapted from Bastos et al., 2001) between the $\mathrm{C}$-terminus of the $1 \mathrm{D}$ capsid proteins of NAM/307/98 and $\mathrm{O}_{1} \mathrm{BFS}$. The $\beta \mathrm{G}$ $\beta \mathrm{H}$ loop RGD sequence is indicated in magenta and the second, non- $\beta \mathrm{G}-\beta \mathrm{H}$ loop RGD, which corresponds to the KAP sequence of $\mathrm{O}_{1} \mathrm{BFS}$, is indicated in red. The predicted secondary structure is presented above the alignment with " $\mathrm{H}$ " $=\alpha$-helix, " $\mathrm{E} "=\beta$-strand and "-" representing connecting loops. (B) Overall three-dimensional structure of the protomeric subunit of $\mathrm{O}_{1} \mathrm{BFS}$ (PDB: 1FOD; Logan et al., 1993), manipulated in PyMOL 
(DeLano Scientific LLCC 2005) with 1D coloured in green, 1B in blue and 1C in yellow. The $\beta \mathrm{G}-\beta \mathrm{H}$ loop cell attachment RGD motif is indicated in magenta sticks whilst the $\mathrm{O}_{1}$ BFS KAP sequence that corresponds to the second $\beta \mathrm{F}-\beta \mathrm{G}$ loop RGD sequence of NAM/307/98 is indicated in red sticks. (C) A cartoon model of the pentamers in the top half of the $\mathrm{O}_{1} \mathrm{BFS}$ capsid (PDB: 1QQP; Fry et al., 1999) showing the location of the KAP sequence (in red), which corresponds to the second RGD sequence in NAM/307/98, surrounding the five-fold axes. This image was made with VMD, owned by the Theoretical and Computational Biophysics Group, an NIH Resource for Macromolecular Modeling and Bioinformatics, at the Beckman Institute, University of Illinois at UrbanaChampaign. The colour schemes for the different capsid proteins are similar to B.

\subsection{Receptor binding properties of the SAT1 type FMDV field isolate NAM/307/98}

Plaque assays in different cell lines were performed to determine whether NAM/307/98 is capable of utilizing integrins and/or heparan sulfate proteoglycans as receptors for attachment to susceptible cells. In contrast to BHK-21 and IB-RS-2 cells, which are permissive for field strains of FMDV (de Castro, 1964 and de la Torre et al., 1985), CHO-K1 cells are non-permissive despite expressing two RGD-binding integrins, $\alpha_{\mathrm{v}} \beta_{5}$ and $\alpha_{5} \beta_{1}$ (Neff and Baxt, 2001 and Jackson et al., 2004). However, CHO-K1 cells are susceptible to infection by FMDV strains that have been adapted for growth in cultured cells and use heparan sulfate as receptors without the mediation of integrins (Sa-Carvalho et al., 1997, Jackson et al., 1997 and Neff et al., 1998). For comparative purposes, ZIM/7/83, a tissue culture-adapted SAT2 type FMDV capable of utilizing heparan sulfate as receptor for cell binding (Van Rensburg et al., 2004), was therefore included in the plaque assays.

Comparison of the plaque morphologies indicated that although both ZIM/7/83 (Fig. 2A) and NAM/307/98 (Fig. 2B) viruses displayed clear large plaques on IB-RS-2 cells, distinct differences were observed on the other tissue culture cell lines. ZIM/7/83 produced large clear plaques on BHK-21 cells, whilst NAM/307/98 produced small turbid plaques, suggesting a slower rate of replication for the latter virus in these cells. In contrast to ZIM/7/83, which produced small clear plaques on $\mathrm{CHO}-\mathrm{K} 1$ cells and is characteristic of viruses using heparan sulfate proteoglycans as cell receptors, 
$\mathrm{NAM} / 307 / 98$ was unable to produce plaques on this cell line. These results therefore strongly implied that NAM/307/98 is capable of using integrins only as cellular receptors.

(A)
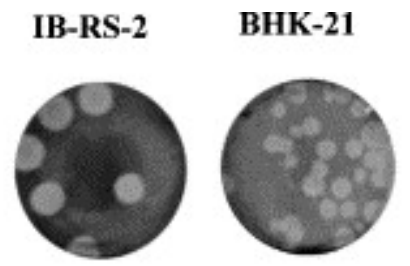

CHO-K1

(B)
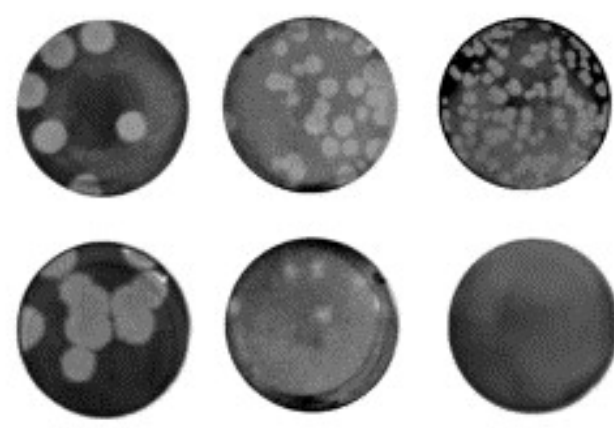

(C)
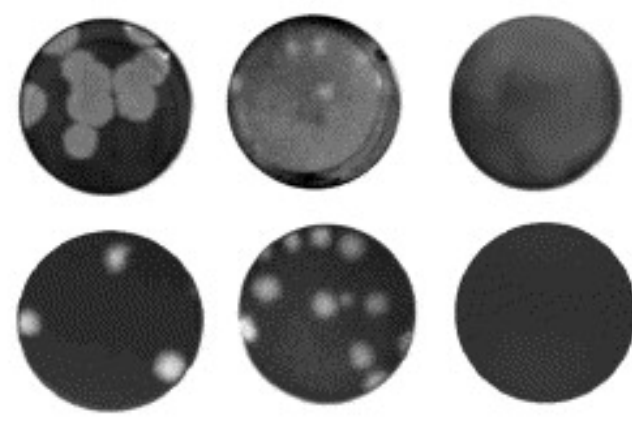

(D)
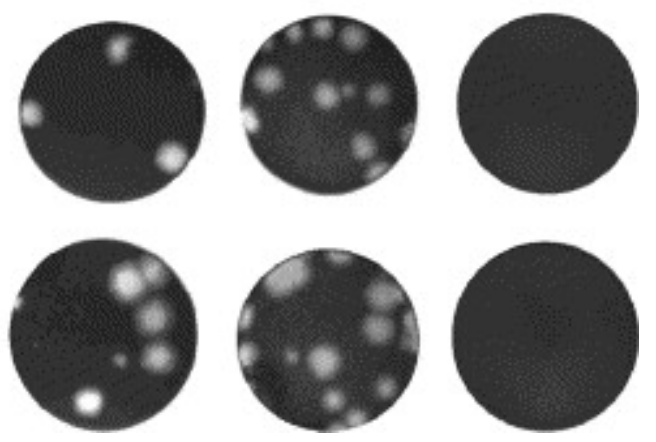

Fig. 2. Plaque morphologies of the tissue culture-adapted SAT2/ZIM/7/83 virus (A), the FMDV field isolate SAT1/NAM/307/98 (B), and the derived genetically engineered viruses vNAM/SAT2 (C) and vNAMRGD/SAT2 (D) on IB-RS-2, BHK-21 and CHO-K1 cells.

\subsection{Infectivity of the chimeric and mutant chimeric NAM/SAT2 viruses}

In order to determine whether the non- $\beta \mathrm{G}-\beta \mathrm{H}$ loop RGD motif plays a role in the ability of NAM/307/98 to bind to BHK-21 cells, cDNA clones pNAM/SAT2, carrying the external capsid-encoding region of NAM/307/98 in the pSAT2 genetic backbone, and pNAMKGE/SAT2, in which the conserved $\beta$ G- $\beta$ H loop RGD sequence of NAM/307/98 was changed to KGE through a PCR-based site-directed mutagenesis strategy, were used as templates for in vitro synthesis of chimeric full-length RNAs that were used to transfect BHK cells. The recovered viruses were passaged four times in BHK cells and the cells were monitored for signs of cytopathic effect (CPE) by comparison to mockinfected cells. The results, shown in Table 1, indicated that BHK cells transfected with 
RNA derived from pSAT2, a genome-length cDNA clone of the highly tissue cultureadapted ZIM/7/83 virus, produced a virus that displayed CPE upon the first round of passage, and is in agreement with results reported previously for the derived vSAT2 virus (Van Rensburg et al., 2004). BHK cells transfected with RNA derived from pNAM/SAT2 produced a virus (vNAM/SAT2) that displayed a clear ability to cause CPE by $\mathrm{BHK}$ passage 4 . In contrast, no CPE was observed during the sequential passaging of the vNAMKGE/SAT2 virus recovered from BHK cells transfected with RNA derived from $\mathrm{pNAMKGE/SAT2}$. These results therefore suggested that the non- $\beta \mathrm{G}-\beta \mathrm{H}$ RGD motif is not capable of mediating attachment of NAM/307/98 to the BHK cells. Table 1.

CPE observed during serial passage of genetically engineered viruses harvested from BHK-21 cells transfected with in vitro synthesized RNA

\begin{tabular}{|l|l|l|l|l|}
\hline \multicolumn{5}{|c|}{ CPE $^{\text {a observed }}$} \\
\hline & BHKp1 & BHKp2 & BHKp3 & BHKp4 \\
\hline Mock-infection & - & - & - & - \\
\hline vSAT2 & ++++ & ++++ & ++++ & ++++ \\
\hline vNAM/SAT2 & - & - & + & ++ \\
\hline vNAMKGE/SAT2 & - & - & - & - \\
\hline vNAMRGD/SAT2 & - & - & - & ++ \\
\hline
\end{tabular}

${ }^{\mathrm{a}} \mathrm{CPE}$, scored by visual observation, was given a value of $(-)$ (no CPE) to $(++++)$ (complete cell destruction).

Further characterization of the apparent inability of the non- $\beta \mathrm{G}-\beta \mathrm{H}$ loop RGD sequence to facilitate binding of the vNAMKGE/SAT2 virus mutant to BHK cells entailed the construction of a second chimera, pNAMRGD/SAT2, in which the KGE mutation was reverted to the wild-type RGD sequence. The results obtained upon transfection of BHK cells with RNA derived from pNAMRGD/SAT2 resembled those obtained for vNAM/SAT2. After an initial lack of CPE, continued passaging of vNAMRGD/SAT2 resulted in CPE being observed by BHK passage 4 (Table 1). 
Plaque assays were performed to verify that the recovered viruses vNAM/SAT2 (Fig. 2C) and vNAMRGD/SAT2 (Fig. 2D) were indeed capable of using integrins as cellular receptors. Both viruses, like the parental NAM/307/98 virus, formed plaques on IB-RS-2 and BHK-21 cells but not on CHO-K1 cells, which are permissive for FMDV strains that use heparan sulfate as receptors, therefore implying the usage of integrin receptors by the chimera and mutant viruses.

\subsection{Detection of viral RNA replication and production of chimeric and mutant chimeric NAM/SAT2 viruses in BHK-21 cells}

Although the non-infectious nature of vNAMKGE/SAT2 may have been due to the introduction of the RGD-to-KGE mutation that abolished its cell-binding capabilities, it was also possible that the RNA synthesized from pNAMKGE/SAT2 could have been non-infectious and thus failed to produce viable virus. Therefore, to determine whether vNAMKGE/SAT2 viral RNA is indeed capable of replicating in BHK-21 cells, viral RNA was extracted from samples prepared from cell-free tissue culture supernatants and cell monolayers used during transfection and first passage, as well as from lysates (supernatants and cells) of cell monolayers used in subsequent passaging. The samples were treated extensively with RNases to degrade residual synthetic RNA transcripts. Thus, the most likely source of RNA available for virus-specific cDNA synthesis would be that of packaged RNA in progeny viral particles (and therefore protected from degradation by the RNase treatment). The extracted viral RNA was reverse transcribed and a 500-bp amplicon, inclusive of the two RGD motifs of NAM/307/98, was PCRamplified and analyzed by agarose gel electrophoresis.

The results indicated that an amplicon of the expected size was obtained from the samples prepared from both the tissue culture supernatant (Fig. 3A) and the cellular fractions (Fig. 3B) of cells transfected with in vitro synthesized RNA derived from the various plasmid constructs. Comparison of the results obtained for samples prepared from the tissue culture supernatant (Fig. 3C) and the cellular fractions (Fig. 3D), following the first passage on BHK cells, yielded similar results but with the notable exception that no amplicon could be detected in instances where the viral RNA was extracted from cells infected with vNAMKGE/SAT2 (Fig. 3D, lane 5). Analysis of samples prepared from the 
lysate of BHK cells used in subsequent virus passaging (BHK passage 2 to 4) yielded an amplicon of the expected size for cells infected with the control vSAT2 virus, the chimeric vNAM/SAT2 virus and KGE-to-RGD reverted vNAMRGD/SAT2 virus. In contrast, no amplicon was obtained from samples prepared from vNAMKGE/SAT2infected BHK cells (Fig. 3E-G). At no stage during these experiments were amplicons produced from samples prepared from mock-infected cells, thus confirming the specificity of the RT-PCR assay for viral RNA only.
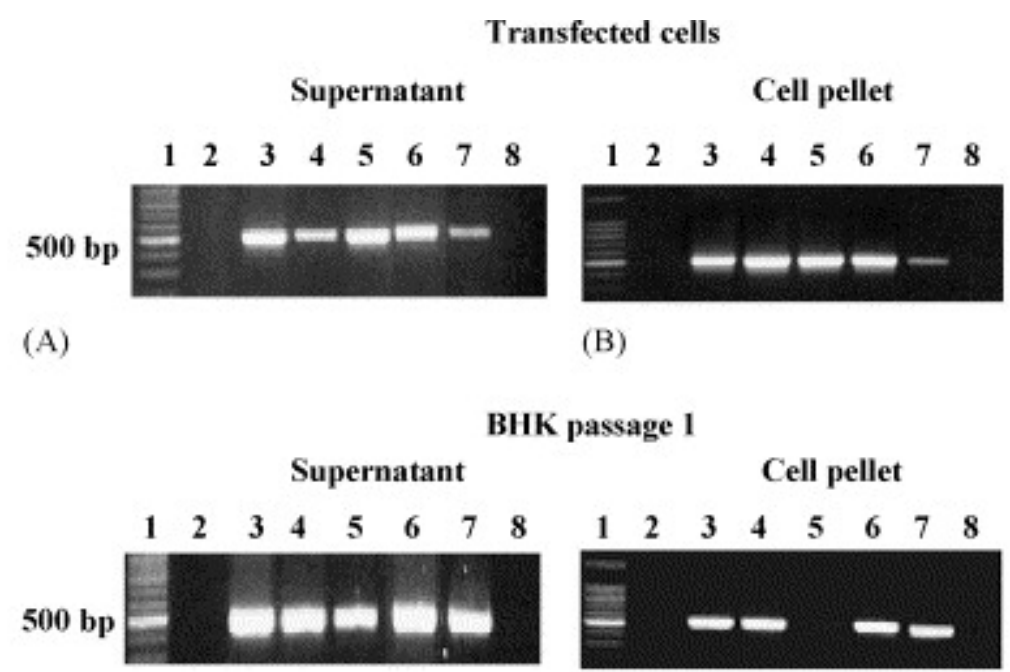

CPE observed: $\quad-\quad-\quad+-$

(C)

(D)

Cell lysate of BHK passages 2-4

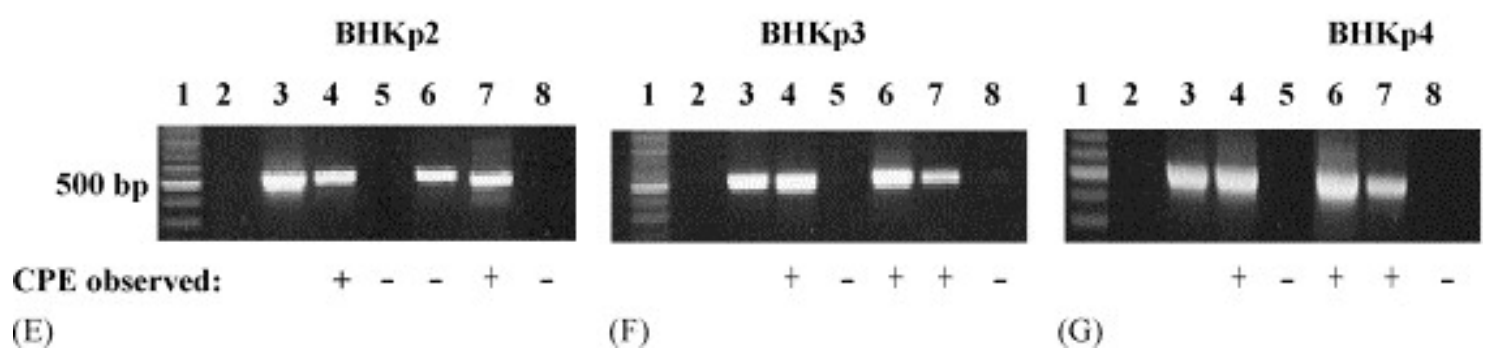

Fig. 3. Detection of viral RNA replication. Samples were prepared from BHK cell monolayers transfected with RNA transcripts derived from the different cDNA clones (panels A and B) and from cell monolayers used for successive passaging of the recovered genetically engineered viruses (panels $\mathrm{C}$ through $\mathrm{G}$ ). Following extensive treatment of the samples with RNases, the viral RNA was isolated, reverse transcribed and a 500-bp product of the capsid protein 1D-encoding region was PCR-amplified. In all 
panels: lane 1-100-bp DNA molecular weight ladder; lane 2-PCR reaction lacking template DNA; lane 3-PCR reaction containing pNAM/SAT2 as template DNA; lanes 4, 5, 6, and 7-amplicons derived from vNAM/SAT2, vNAMKGE/SAT2, vNAMRGD/SAT2 and vSAT2, respectively. Mock-infected cells (lane 8) were treated identically and included as a control in these assays. The CPE, observed visually, following each round of virus passage is indicated below the panels. Symbols: (-) no CPE observed; (+) CPE observed $48 \mathrm{~h}$ after infection.

Furthermore, to determine whether the recovered viruses had maintained the cDNAencoded sequence, the nucleotide sequence was determined of the amplicons obtained from samples prepared from the tissue culture supernatant of transfected cells and of the lysates prepared from the highest passaged cells. Analysis of the sequence data revealed that viruses derived from cDNA clones harbouring the external capsid-encoding region of NAM/307/98 (vNAM/SAT2, vNAMKGE/SAT2, vNAMRGD/SAT2) faithfully maintained the sequence encoded by the original chimeric DNA in cell culture (Fig. 4).

$\mathrm{SAT} 1 / \mathrm{NAM} / 307 / 98$ VNAM/SAT2 VNAMKGE/SAT2 VNAMRGD/SAT2 (A)

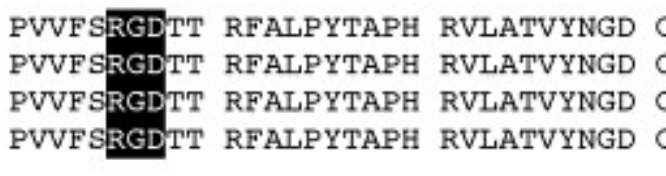
PVVFSRGDTT RFALPYTAPH RVLATVYNGD
SAT1/NAM/ 307/98 vNAM/SAT2 VNAMRGD/SAT2

(B)
PVVFSRGDTT RFALPYTAPH RVLATVYNGD CKYKPTGTPP RENIRGDLAT LAARIASETH PVVFSRGDTT RFALPYTAPH RVLATVYNGD CKYKPTGTPP RENIRGDLAT LAARIASETH PVVFSRGDTT RFALPYTAPH RVLATVYNGD CKYKPTGTPP, RENIRGTAT LAARIASETH $\beta G-\beta H$ loop

Fig. 4. Amino acid sequence alignment of the partial 1D capsid protein of SAT1/NAM/307/98, chimeric vNAM/SAT2, vNAMKGE/SAT2 and vNAMRGD/SAT2 viruses. The deduced amino acid sequence of the partially sequenced 1D capsid-encoding region from amplicons derived from samples prepared from (A) the supernatant of transfected BHK-21 cells and (B) lysates prepared from the highest passage cells (BHKp4) is presented. The $\beta \mathrm{G}-\beta \mathrm{H}$ loop RGD sequence is indicated in bold, whilst a second, upstream RGD motif is highlighted in inverted text. Substitution of the $\beta \mathrm{G}-\beta \mathrm{H}$ loop RGD sequence with a KGE sequence (vNAMKGE/SAT2) is highlighted in gray, 
whilst substitution of the KGE sequence with an RGD sequence (vNAMRGD/SAT2) is indicated in italics. The $\beta \mathrm{G}-\beta \mathrm{H}$ loop is indicated by asterisk $\left(^{*}\right)$, corresponding to amino acid residues 136-162 of the 1D capsid protein (numbering according to Bastos et al., 2001).

The presence of viral particles resulting from transfection with mutant and non-mutant chimeric RNAs were verified by electron microscopy, following isolation of chimeric virus particles from the transfected cells (Fig. 5). Virus particles were observed for the chimeric viruses with either an infectious phenotype (vNAM/SAT2 (A) and vNAMRGD/SAT2 (B)) or non-infectious phenotype (vNAMKGE/SAT2 (C)). Through RT-PCR it was possible to establish that these capsids contain packaged RNA (results not shown). These findings confirmed that the $\beta \mathrm{G}-\beta \mathrm{H}$ loop RGD motif is necessary for the cell attachment of NAM/307/98. 
(A)
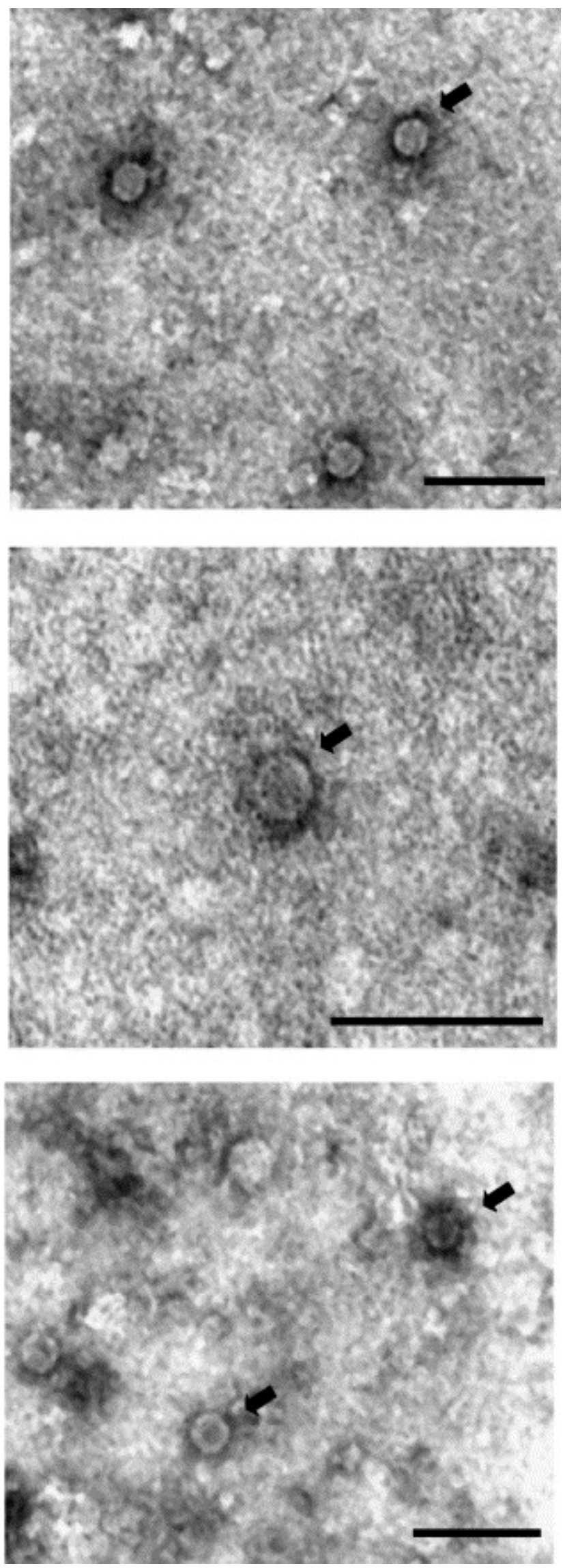
Fig. 5. Electron microscopy of infectious and non-infectious genetically engineered chimeric viruses. Cells were harvested $24 \mathrm{~h}$ after transfection with in vitro synthesized RNA transcripts and virus particles were isolated by sucrose density gradient centrifugation and stained with uranyl acetate. (A) vNAM/SAT2, (B) vNAMRGD/SAT2 and $(\mathrm{C}) \mathrm{vNAMKGE} / \mathrm{SAT} 2$. Bar $=100 \mathrm{~nm}$.

\section{Discussion}

Sequence analysis of the 1D-encoding region of a SAT1 FMDV field isolate, $\mathrm{NAM} / 307 / 98$, indicated the presence of two RGD motifs, both of which were found to be located on surface-exposed loops (Fig. 1). Moreover, the isolate was shown to utilize a RGD-dependent receptor as evidenced by plaques on BHK-21 and IB-RS-2 cells only (Fig. 2). In this study we investigated the functional importance of these RGD sequences to mediate attachment of NAM/307/98 to susceptible cells. Using a reverse genetics approach, chimeric viruses were engineered using a SAT2/ZIM/7/83 infectious cDNA (Van Rensburg et al., 2004) and the external capsid protein-encoding region of SAT1/NAM/307/98. The conserved $\beta$ G- $\beta$ H loop RGD sequence in capsid protein $1 D$ was mutated to KGE, thus leaving the second, upstream RGD sequence intact. Not only did the KGE-for-RGD substitution maintain the "positive-glycine-negative" charge motif of the wild-type RGD sequence, but this mutation has been reported to yield FMD mutant viruses that were able to bind to a panel of monoclonal antibodies (Mason et al., 1994). Therefore, this mutation was not expected to induce extensive rearrangements in the capsid protein 1D. Hence, it was expected that the function of the NAM/307/98 1D protein, except that of the $\beta \mathrm{G}-\beta \mathrm{H}$ loop RGD motif itself, would be maintained. Substitution of the $\beta G-\beta H$ loop RGD sequence with a KGE tripeptide had a pronounced effect on viral replication. Although cells transfected with the full-length mutant transcripts showed CPE, no cell lysis could be observed upon subsequent passaging of the produced vNAMKGE/SAT2 virus, despite the presence of viral particles. In contrast, vNAMRGD/SAT2, which contained a RGD-for-KGE substitution, was fully infectious and resembled vNAM/SAT2, which contained the wild-type external capsid-encoding region. Since plaque assays on different cell lines confirmed that both vNAM/SAT2 and vNAMRGD/SAT2 do not utilize a non-RGD-dependent alternative receptor, these results 
indicated that the $\beta \mathrm{G}-\beta \mathrm{H}$ loop RGD sequence, but not the second RGD motif, mediates attachment of the virus to BHK-21 cells. Notably, CPE observed during the sequential passaging of these chimeric viruses lagged behind vSAT2. This might have been due to the initial viral isolation of the parental NAM/307/98, and therefore adaptation of the virus, to pig kidney cell lines (primary pig kidney and IB-RS-2). Since the cell binding properties of the parental virus have been transferred to the genetically engineered viruses as suggested by the plaque assays (Fig. 2), the slow recovery of these viruses following RNA transfection of BHK-21 cells (Table 1) could reflect the subsequent adaptation of the chimeric viruses to this cell line.

Previously, non-infectious viral particles were also obtained for a FMDV type A virus where the $\beta \mathrm{G}-\beta \mathrm{H}$ loop RGD sequence was changed for a KGE triplet (Mason et al., 1994). However, it has been shown that FMDV type O virus strains that contained a KGE sequence in place of the naturally occurring integrin-binding RGD are able to replicate well in cell culture (Zhao et al., 2003), whilst type C viruses have been reported to be capable of initiating infection in the absence of the highly conserved RGD motif (Martinez et al., 1997 and Baranowski et al., 2000). In this study, evidence was obtained indicating RNA replication and virus assembly of the genetically engineered vNAMKGE/SAT2 virus, despite its non-infectious phenotype. In contrast to the above tissue culture-passaged FMDV strains, these results therefore confirmed that the $\beta \mathrm{G}-\beta \mathrm{H}$ loop RGD motif of the NAM/307/98 field isolate is functionally relevant and conversely, that the second RGD motif does not function as a receptor-binding site for infection of BHK-21 cells.

In addition to the $\beta \mathrm{G}-\beta \mathrm{H}$ loop RGD motif, it has been reported that sequences following this motif may also be important for receptor binding (Jackson et al., 2000a). Despite its ability to use different integrin receptors, field isolates of FMDV have been shown to use RGD-dependent integrin $\alpha_{\mathrm{v}} \beta_{3}$ as a cellular receptor on cultured cells (Berinstein et al., 1995, Jackson et al., 1996 and Neff et al., 1998). Integrin $\alpha_{v} \beta_{3}$ is a multifunctional receptor and binds a broad range of RGD-containing ligands (Hynes, 1992). Consistent with this role, ligand binding by $\alpha_{v} \beta_{3}$ has been shown to tolerate several different amino acids flanking the RGD, including those of the RGD +1 and RGD +4 positions (Healy et al., 1995). Therefore, the inability of vNAMKGE/SAT2 to initiate subsequent rounds 
of infection, despite the presence of a second, intact RGD motif, may more likely be as a consequence of its location on the virion surface. The long, mobile $\beta \mathrm{G}-\beta \mathrm{H}$ loop may present the RGD motif, located at its apex, in a conformation appropriate for direct interaction of the oppositely orientated Arg (R) and Asp (D) side chains with the cell receptors (D'Souza et al., 1991, Mateu et al., 1996 and Domingo et al., 1999). As shown in Fig. 1, the non- $\beta$ G- $\beta$ H loop RGD sequence of NAM/307/98 is located on a short surface-exposed loop. It is therefore possible that this short loop might not possess the flexibility required to present the RGD motif in such a way as to allow for interaction with the cellular receptor.

In conclusion, our study reveals that the $\beta G-\beta H$ loop RGD-mediated virus-cell interaction appears to be specific and relevant for the SAT1 type field isolate $\mathrm{NAM} / 307 / 98$. This conclusion is also supported by the finding that destruction of the $\beta \mathrm{G}-$ $\beta \mathrm{H}$ loop RGD motif by site-directed mutagenesis yielded viruses capable of replication and virus assembly, but were unable to initiate further cycles of infection due to the inability to interact with the cellular receptor. Since no productive infection could be achieved, despite the presence of an intact second RGD motif, the $\beta G-\beta H$ loop RGDmediated cell recognition appears to be the only means for attachment of NAM/307/98 to BHK-21 cells.

\section{References}

Acharya et al., 1989 R. Acharya, E. Fry, D. Stuart, G. Fox, D. Rowlands and F. Brown, The three-dimensional structure of foot-and-mouth disease virus at $2.9 \AA$ resolution, Nature 337 (1989), pp. 709-716.

Acharya et al., 1990 R. Acharya, E. Fry, D. Stuart, G. Fox, D. Rowlands and F. Brown, The structure of foot-and-mouth disease virus: implications for its physical and biological properties, Vet. Microbiol. 23 (1990), pp. 21-34.

Baranowski et al., 2000 E. Baranowski, C.M. Ruiz-Jarabo, N. Sevillia, D. Andreu, E. Beck and E. Domingo, Cell recognition by foot-and-mouth disease virus that lacks the RGD integrin-binding motif: flexibility in Apthovirus receptor usage, J. Virol. 74 (2000), pp. 1641-1647. 
Bastos, 1998 A.D.S. Bastos, Detection and characterization of the foot-and-mouth disease virus in sub-Saharan Africa, Onderstepoort J. Vet. Res. 65 (1998), pp. 37-47. Bastos et al., 2001 A.D.S. Bastos, D. Haydon, R. Forsberg, N. Knowels, E. Anderson, R. Bengis, L.H. Nel and G.R. Thomson, Genetic heterogeneity of SAT-1 type foot-andmouth disease viruses in southern Africa, Arch. Virol. 146 (2001), pp. 1537-1551. Baxt and Becker, 1990 B. Baxt and Y. Becker, The effect of peptides containing the arginine-glycine-aspartic acid sequence on the adsorption of foot-and-mouth disease virus to tissue culture cells, Virus Genes 4 (1990), pp. 73-83.

Baxt and Mason, 1995 B. Baxt and P.W. Mason, Foot-and-mouth disease virus undergoes restricted replication in macrophage cell cultures following Fc receptormediated adsorption, Virology 207 (1995), pp. 503-509.

Berinstein et al., 1995 A. Berinstein, M. Roivainen, T. Hovi, P.W. Mason and B. Baxt, Antibodies to the vitronectin receptor (integrin $\alpha_{v} \beta_{3}$ ) inhibit binding and infection of footand-mouth disease virus to cultured cells, J. Virol. 69 (1995), pp. 2664-2666.

Curry et al., 1995 S. Curry, C.C. Abrams, E. Fry, J.C. Crowther, G.J. Belsham, D.I. Stuart and A.M.Q. King, Viral RNA modulates the acid sensitivity of foot-and-mouth disease virus capsids, J. Virol. 69 (1995), pp. 430-438. de Castro, 1964 M.P. de Castro, Behaviour of foot-and-mouth disease virus in cell cultures. Susceptibility of IB-RS-2 cell line, Archiv. Inst. Biol. Sao Paulo 3 (1964), pp. $63-78$.

de la Torre et al., 1985 J.C. de la Torre, M. Da Villa, F. Sobrino, J. Ortin and E. Domingo, Establishment of cell lines persistently infected with foot-and-mouth disease virus, Virology 145 (1985), pp. 24-35.

Domingo et al., 1999 E. Domingo, N. Verdaguer, W.F. Ochoa, C.M. Ruiz-Jarabo, N. Sevilla, E. Baranowski, M.G. Mateu and I. Fita, Biochemical and structural studies with neutralizing antibodies raised against foot-and-mouth disease virus, Virus Res. 62 (1999), pp. 169-175.

D'Souza et al., 1991 S. D'Souza, M. Ginsberg and E. Plow, Arginyl-glycyl-aspartic acid (RGD): a cell adhesion motif, TiBS 16 (1991), pp. 246-250. 
Fox et al., 1989 G. Fox, N.R. Parry, P.V. Barnett, B. McGinn, D.J. Rowlands and F. Brown, The cell attachment site on foot-and-mouth disease virus includes the amino acid sequence RGD (arginine-glycine-aspartic acid), J. Gen. Virol. 70 (1989), pp. 625-637. Fry et al., 1990 E. Fry, D. Logan, R. Acharya, G. Fox, D. Rowlands, F. Brown and D. Stuart, Architecture and topography of an aphthovirus, Semin. Virol. 1 (1990), pp. 439451.

Fry et al., 1999 E.E. Fry, S.M. Lea, T. Jackson, J.W. Newman, F.M. Ellard, W.E. Blakemore, R. Abu-Ghazaleh, A. Samuel, A.M. King and D.I. Stuart, The structure and function of a foot-and-mouth disease virus-oligosaccharide receptor complex, EMBO J. 18 (1999), pp. 543-554.

Grubman et al., 1979 M.J. Grubman, B. Baxt and H.L. Bachrach, Foot-and-mouth disease virion RNA: studies on the relation between the length of its 3'-poly(A) segment and infectivity, Virology 97 (1979), pp. 22-31.

Grubman and Baxt, 2004 M. Grubman and B. Baxt, Foot-and-mouth disease, Clin. Microbiol. Rev. 17 (2004), pp. 465-493.

Healy et al., 1995 J.M. Healy, O. Murayama, T. Maeda, K. Yoshino, K. Sekiguchi and M. Kikuchi, Peptide ligands for integrin $\alpha_{v} \beta_{3}$ selected from random phage display libraries, Biochemistry 34 (1995), pp. 3948-3955.

Hynes, 1992 R.O. Hynes, Integrins: versatility, modulation and signaling in cell adhesion, Cell 69 (1992), pp. 11-25.

Jackson et al., 1996 T. Jackson, F.M. Ellard, R. Abu-Ghazaleh, S.M. Brookes, W.E. Blakemore, A.H. Corteyn, D.L. Stuart, J.W.I. Newman and A.M.Q. King, Efficient infection of cells in culture by type $\mathrm{O}$ foot-and-mouth disease virus requires binding to cell surface heparan sulfate, J. Virol. 70 (1996), pp. 5285-5287.

Jackson et al., 1997 T. Jackson, A. Sharma, R. Abu-Ghazaleh, W. Blakemore, E. Ellard, D.L. Simmons, D.I. Stuart, J.W.I. Newman and A.M.Q. King, Arginine-glycine-aspartic acid-specific binding by foot-and-mouth disease viruses to the purified integrin $\alpha_{v} \beta_{3}$ in vitro, J. Virol. 71 (1997), pp. 8357-8361.

Jackson et al., 2000a T. Jackson, W. Blakemore, J.W. Newman, N.J. Knowles, A.P. Mould, M.J. Humphries and A.M. King, Foot-and-mouth disease virus is a ligand for the high-affinity binding conformation of integrin $\alpha_{5} \beta_{1}$ : influence of the leucine residue 
within the RGDL motif on selectivity of integrin binding, J. Gen. Virol. 81 (2000), pp. 1383-1391.

Jackson et al., 2000b T. Jackson, D. Sheppard, M. Denyer, W. Blakemore and A.M.Q. King, The epithelial integrin $\alpha_{v} \beta_{6}$ is a receptor for foot-and-mouth disease virus, J. Virol. 74 (2000), pp. 4949-4956.

Jackson et al., 2002 T. Jackson, A.P. Mould, D. Sheppard and A.M.Q. King, Integrin $\alpha_{\mathrm{v}} \beta_{1}$ is a receptor for foot-and-mouth disease virus, J. Virol. 76 (2002), pp. 935-941. Jackson et al., 2004 T. Jackson, S. Clark, S. Berryman, A. Burman, S. Cambier, D. Mu, S. Nishimura and A.M.Q. King, Integrin $\alpha_{\mathrm{v}} \beta_{8}$ functions as a receptor for foot-and-mouth disease virus: role of the $\beta$-chain cytodomain in integrin-mediated infection, J. Virol. 78 (2004), pp. 4533-4540.

Landt et al., 1990 O. Landt, H.P. Grunert and U. Hahn, A general method for rapid sitedirected mutagenesis using the polymerase chain reaction, Gene 96 (1990), pp. 125-128. Logan et al., 1993 D. Logan, R. Abu-Ghazaleh, W. Blakemore, S. Curry, T. Jackson, A.M.Q. King, S. Lea, R. Lewis, J. Newman, N. Parry, D. Rowlands, D. Stuart and E. Fry, Structure of a major immunogenic site on foot-and-mouth disease virus, Nature 362 (1993), pp. 566-568.

Martinez et al., 1997 M.A. Martinez, N. Verdaguer, M.G. Mateu and E. Domingo, Evolution subverting essentiality: dispensability of the cell attachment Arg-Gly-Asp motif in multiply passaged foot-and-mouth disease virus, Proc. Natl. Acad. Sci. U.S.A. 94 (1997), pp. 6798-6802.

Mason et al., 1993 P.W. Mason, B. Baxt, F. Brown, J. Harber, A. Murdin and E. Wimmer, Antibody-complexed foot-and-mouth disease virus, but not poliovirus can infect normally insusceptible cells via the Fc receptor, Virology 192 (1993), pp. 568-577. Mason et al., 1994 P.W. Mason, E. Rieder and B. Baxt, RGD sequence of foot-andmouth disease is essential for infecting cells via the natural receptor but can be bypassed by an antibody-dependent enhancement pathway, Proc. Natl. Acad. Sci. U.S.A. 91 (1994), pp. 1932-1936.

Mateu, 1995 M.G. Mateu, Antibody recognition of picornaviruses and escape from neutralization: a structural view, Virus Res. 38 (1995), pp. 1-24. 
Mateu et al., 1996 M.G. Mateu, M. Luz Valero, D. Andreu and E. Domingo, Systematic replacement of amino acid residues within an Arg-Gly-Asp containing loop of foot-andmouth disease virus and effects on cell recognition, J. Biol. Chem. 271 (1996), pp. $12814-12819$.

Neff et al., 1998 S. Neff, D. Sa-Carvalho, E. Rieder, P.W. Mason, S.D. Blystone, E.J. Brown and B. Baxt, Foot-and-mouth disease virus virulent for cattle utilizes the integrin $\alpha_{\mathrm{v}} \beta_{3}$ as its receptor, J. Virol. 72 (1998), pp. 3587-3594.

Neff and Baxt, $2001 \mathrm{~S}$. Neff and B. Baxt, The ability of integrin $\alpha_{\mathrm{v}} \beta_{3}$ to function as a receptor for foot-and-mouth disease virus is not dependent on the presence of the complete subunit cytoplasmic domain, J. Virol. 75 (2001), pp. 527-532.

Parry et al., 1990 N. Parry, G. Fox, D. Rowlands, F. Brown, E. Fry, R. Acharya and D. Stuart, Structural and serological evidence for a novel mechanism of antigenic variation in foot-and-mouth disease virus, Nature 347 (1990), pp. 569-572.

Sa-Carvalho et al., 1997 D. Sa-Carvalho, E. Rieder, B. Baxt, R. Rodarte, T. Tanuri and P.W. Mason, Tissue culture adaptation of foot-and-mouth disease viruses that bind to heparin and are attenuated in cattle, J. Virol. 71 (1997), pp. 5115-5123.

Thomson et al., 2003 G.R. Thomson, W. Vosloo and A.D. Bastos, Foot-and-mouth disease in wildlife, Virus Res. 91 (2003), pp. 145-161.

Thomson and Bastos, 2004 G.R. Thomson and A.D.S. Bastos, Foot-and-mouth disease. In: J.A.W. Coetzer and R.C. Tustin, Editors, Infectious Diseases of Livestock, Oxford University Press, Cape Town (2004), pp. 1323-1365.

Vangrysperre and De Clercq, 1996 W. Vangrysperre and K. De Clercq, Rapid and sensitive polymerase chain reaction-based detection and typing of foot-and-mouth disease virus in clinical samples and cell culture isolates, combined with a simultaneous differentiation with other genomically and/or symptomatically related viruses, Arch. Virol. 141 (1996), pp. 331-344.

Van Rensburg et al., 2004 H.G. Van Rensburg, T.M. Henry and P.W. Mason, Studies of genetically defined chimeras of a European type A virus and a South African territories type 2 virus reveal growth determinants for foot-and-mouth disease virus, J. Gen. Virol. 85 (2004), pp. 61-68. 
Zhao et al., 2003 Q. Zhao, J. Pacheco and P.W. Mason, Evaluation of genetically engineered derivatives of a Chinese strain of foot-and-mouth disease reveals a novel cellbinding site which functions in cell culture and in animals, J. Virol. 77 (2003), pp. 32693280.

Corresponding author. Present address: Division of Medical Biochemistry, Institute of Infectious Diseases and Molecular Medicine, University of Cape Town, Observatory 7925, South Africa. Tel.: +27 21406 6814; fax: +27 214066061 . 